\title{
Pseudo-PID Control of Pulse Width Modulation Inverter for Relay Testing
}

\author{
Xiaoming Sun \\ Chongqing Water Resources and Electric Engineering College, Chongqing, Yongchuan 402160, China
}

\begin{abstract}
P}$ and PI control methods are widely used in the closed-loop control of the inverters in various power electronic equipment. However, PID control method is barely adopted. In this paper, from an in-depth analysis of the operation and control processes of pulse width modulation (PWM) inverter, it is verified that PID control method is not suitable for inverter control. And the theoretical process gives birth to a new control method, which is similar but not identical to PID control method, therefore, it is named as pseudo-PID control method. For fundamentality and generality, the pseudo-PID control method is derived from the single-phase PWM inverter, which is the basis of threephase or multilevel inverters. To verify the performance of the pseudo-PID control method, it is applied to a single-phase PWM inverter for relay testing. The simulation and experimental results demonstrate that with the aid of pseudo-PID control method the accuracy of the output current of the inverter is already comparable to that of the linear power amplifier (LPA), which makes the application of inverter in relay testing feasible. Considering the higher efficiency and simpler structure of the PWM inverter, it can make the relay testing device more cost-effective.
\end{abstract}

Keywords-pseudo-PID control; pulse width modulation; single-phase inverter; relay testing

\section{INTRODUCTION}

Because of the technical maturity, P and PI control methods are widely used in the closed-loop control of the inverters in various power electronic equipment, controlling the whole system or the local current or voltage [1-4]. And, the high execution speed, the quick current response and the constant switching frequency of the switching devices are their prominent advantages, especially for real-time tracking purpose. However, their counterpart, i.e., PID control method, is barely adopted; the report of the successful application of PID control method in the closed-loop control of inverters can hardly be found in authoritative journals. In fact, the empirical research of the authors indicates that the introduction of $\mathrm{D}$ parameter will bring about the following two problems.

Firstly, the tuning of PID parameters becomes more complex. Historically, PID control method originated from the classic control theory that can only deal with the continuous analog systems with minimum phase effectively [5]. However, the inverter is a special system consisting of some discrete digital elements and several pure time delay elements. Therefore, unlike the continuous analog systems with mini-mum phase, it is impossible to obtain the detailed formulae from the classic control theory to tune the PID parameters directly. Thus, the practical way is to tune the PID parameters through trial and error with empirical experience. How-ever, considering the different functions and effects of each parameter and their combinations, only tuning P or PI parameters is sometimes time-consuming, when D parameter is added in, the workload is greatly increased. And even if a set of applicable parameters is obtained, the optimality can still not be guaranteed. Perhaps, the intelligent control methods can help solve the problem. However, the fuzzy PID control method and the expert PID control method [6-8] depend highly on the rule base and the knowledge base of the PID parameters, which are also constructed on the basis of plentiful empirical experiences. And, although many adaptive methods (e.g. the robust adaptive PID control method [9], the artificial neural network PID control method [10], the particle swarm PID control method [11], the evolutionary PID control method [12] and the genetic PID control method [13], etc.) can automatically tune the PID parameters online, the process entails a large quantity of computations, raising a high demand upon the microprocessor and thus being un-suitable for realtime control.

Secondly, the stability and reliability of the system are likely to degrade. When the inverter is operating stably and reliably under P or PI control method, the introduction of D parameter will sometimes cause oscillations, or sometimes seem to have no effect on the operation of the system. And this is obviously undesirable during the continuous operation of the system, and is also a problem that can not be solved by the intelligent control methods.

In short, as for the closed-loop control of inverters, abandoning D parameter seems to be a good way to avoid the unnecessary troubles above. However, the crux of the problem is not to avoid it but to figure out, based on theoretical analysis, whether D parameter can be added in or not. Aiming at this, an in-depth analysis of the operation and control processes of the pulse width modulation (PWM) inverter is carried out in this paper. And the analytical result verifies that PID control method is actually unsuitable for the closed-loop control of PWM inverters. Besides, the analytical process has produced three useful formulae, of which the first one is used to calculate $\mathrm{P}$ parameter, the second one is used to calculate I parameter, and the third one is used to calculate a new control term. Because the result of the third formula is not a D parameter, the combination of the three formulae gives birth to a new control method that is similar but not identical to PID control method, and therefore it is named as pseudo-PID control method. To ensure the fundamentality and generality of the discussions, the pseudo-PID control method is derived from the single-phase PWM inverter, which is the basis of the three-phase or multilevel inverters, and thus the pseudo-PID control method can be transplanted to these complex inverters with slight modifications. 
As an application example, the proposed pseudo-PID control method is applied to a single-phase PWM inverter for relay testing to verify its performance. And the simulation and experimental results demonstrate that with the aid of the pseudo-PID control method the accuracy of the output current of the inverter is already comparable to that of the linear power amplifier (LPA), meaning that based on this method the inverter can now replace the LPA for relay testing. And due to the higher efficiency and simpler structure of the PWM inverter, it can make the relay testing device more cost effective.

\section{ANALYSIS OF OPERATION AND CONTROL PROCESSES}

To begin with, an in-depth analysis of the operation and control processes of the single-phase PWM inverter is carried out in this section. The general topology of the single-phase PWM inverter is presented in Figure 1, which is a volt-age source full bridge type inverter, and its basic control principle is also shown in the figure. Here, the snubber circuits are omitted for clarity, the design of which is discussed in [14].

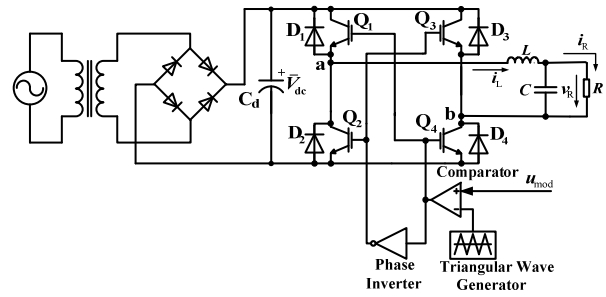

FIGURE I. SINGLE-PHASE PWM INVERTER AND ITS CONTROL PRINCIPLE

For theoretical analysis, the single-phase PWM inverter is modeled by the source period averaging model [15-16], which is one of the multilayer models of the power electronic circuit. The reason to choose this model is that it is very suitable for studying the dynamic and steady-state responses of the system, which are exactly the emphases of this paper. As shown in Figure 1 , the inverter is powered by an AC source together with a rectifier transformer and an uncontrolled rectifier bridge. In operation, the DC voltage of the electrolytic capacitor Cd (denoted as Vdc) will fluctuate constantly with the charging and discharging of $\mathrm{Cd}$, and the main idea of the source period averaging model is to regard the average value of $\mathrm{Vdc}$ (denoted as $\bar{V}_{\mathrm{dc}}$ ) over 1 period of the AC source as a constant. And based on this premise, the Kirchhoff voltage and current equations of the inverter can be listed out:

$$
\left\{\begin{array}{l}
(2 p-1) \bar{V}_{\mathrm{dc}}=L \frac{\mathrm{d} i_{\mathrm{L}}}{\mathrm{d} t}+i_{\mathrm{L}} r+v_{\mathrm{R}}, \\
i_{\mathrm{L}}=C \frac{\mathrm{d} v_{\mathrm{R}}}{\mathrm{d} t}+i_{\mathrm{R}}=R C \frac{\mathrm{d} i_{\mathrm{R}}}{\mathrm{d} t}+i_{\mathrm{R}},
\end{array}\right.
$$

where $L$ and $C$ are the inductance and capacitance of the LC output filter, $R$ is the resistance of the load, $i_{\mathrm{L}}$ is the current of the inductor, $i_{\mathrm{R}}$ is the current of the load, $v_{\mathrm{R}}=i_{\mathrm{R}} R$ is the voltage of the load, $r=r_{\mathrm{Q}}+r_{\mathrm{L}}$ is the total additional resistance of the loop (here, $r_{\mathrm{Q}}$ is the equivalent resistance of the switching de- vices and $r_{\mathrm{L}}$ is the winding resistance of the inductor), and $p$ is a unipolar two-valued logic function

$$
p= \begin{cases}1 & \left(\begin{array}{l}
\mathrm{Q}_{1} \text { and } \mathrm{Q}_{4} \mathrm{ON} \text { or } \mathrm{D}_{1} \text { and } \mathrm{D}_{4} \mathrm{ON} \\
\mathrm{Q}_{2} \text { and } \mathrm{Q}_{3} \mathrm{OFF} \text { and } \mathrm{D}_{2} \text { and } \mathrm{D}_{3} \mathrm{OFF}
\end{array}\right) \\
0 & \left(\begin{array}{l}
\mathrm{Q}_{2} \text { and } \mathrm{Q}_{3} \mathrm{ON} \text { or } \mathrm{D}_{2} \text { and } \mathrm{D}_{3} \mathrm{ON} \\
\mathrm{Q}_{1} \text { and } \mathrm{Q}_{4} \mathrm{OFF} \text { and } \mathrm{D}_{1} \text { and } \mathrm{D}_{4} \mathrm{OFF}
\end{array}\right)\end{cases}
$$

where Q1 Q4 are the insulated-gate bipolar transistors (IGBTs), D1 D4 are the fast-recovery free-wheeling diodes, and "ON" and "OFF" denote "turn on" and "turn off".

Next, to make the inverter start to operate, the PWM signals are needed to trigger the IGBTs. The PWM signals are produced by the triangular carrier comparison procedure in terms of the pulse area equivalent principle, as is depicted in Figure 2. The essence is to use the isosceles triangular carrier to cut, i.e., to sample, the modulation signal umod, and this will yield a curved-edge trapezoidal pulse $\mathrm{ABCDE}$. Besides, the pulse area equivalent principle requires that the area of the curved-edge trapezoidal pulse $\mathrm{ABCDE}$ should be equal to the net area of the PWM signals. Because the sampling period Ts is generally very small, the change of umod over 1 Ts is relatively smooth, and thus the area of the curved-edge trapezoidal pulse ABCDE is close to the area of the rectangle $\mathrm{A}^{\prime} \mathrm{C}^{\prime} \mathrm{DE}$, i.e., the shadowed area SA. And SA can be easily calculated:

$$
S_{\mathrm{A}}=u_{\text {modc }} T_{\mathrm{s}},
$$

where umode is the ordinate of the midpoint B of the arc A-C. Then, denote the net area of the PWM signals as SB, and

$$
S_{\mathrm{B}}=S_{\mathrm{B} 1}-S_{\mathrm{B} 2}-S_{\mathrm{B} 3}=\bar{V}_{\mathrm{dc}} t_{\mathrm{on}}-\bar{V}_{\mathrm{dc}} t_{\mathrm{off}}-\bar{V}_{\mathrm{dc}} t_{\mathrm{off}}
$$

where ton $\in[0, \mathrm{Ts}]$ and toff $\in[0, \mathrm{Ts} / 2]$ are the turn-on time and turn-off time of the two diagonal switching devices. Let $\mathrm{SA}=\mathrm{SB}$, thus umodc can be re-expressed as

$$
u_{\text {modc }}=\left(t_{\text {on }}-\frac{T_{\mathrm{s}}}{2}\right) \frac{2 \bar{V}_{\mathrm{dc}}}{T_{\mathrm{s}}}
$$




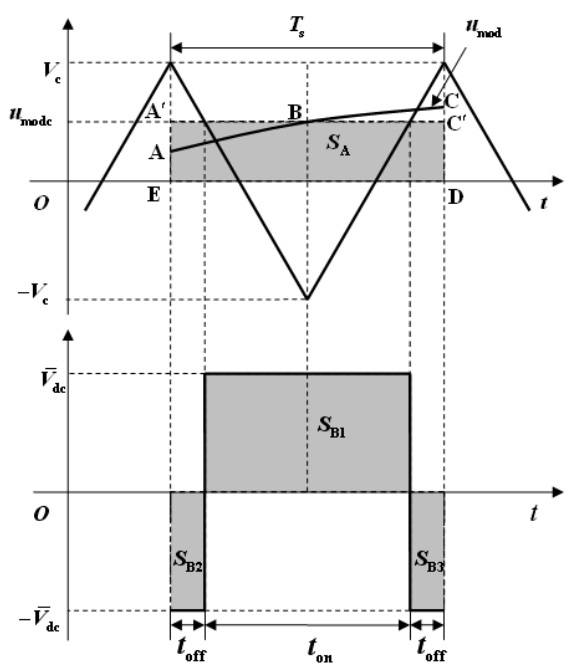

FIGURE II. PWM SIGNALS PRODUCED BY TRIANGULAR CARRIER COMPARISON PROCEDURE

where the default relation $2 t_{\mathrm{off}}=T_{\mathrm{s}}-t_{\mathrm{on}}$ is also involved in the calculation. Here, it should be pointed out that $u_{\text {modc }}$ is not only a geometrical quantity but also a physical quantity, because it is equal to the average voltage between the points $\mathbf{a}$ and $\mathbf{b}$ of Fig. 1. And this can be discovered from the following computations of (5): when $t_{\mathrm{on}}=0$ (i.e., the positive area is 0 and the negative area reaches the maximum), $u_{\text {modc }}=-\bar{V}_{\mathrm{dc}}$; when $t_{\mathrm{on}}=T_{\mathrm{s}} / 2$ (i.e., the positive area is equal to the negative area), $u_{\text {modc }}=0$; when $t_{\mathrm{on}}=T_{\mathrm{s}}$ (i.e., the positive area reaches the maximum and the negative area is 0$), u_{\text {modc }}=\bar{V}_{\mathrm{dc}}$. From this, it is apparent that the switching process of the inverter is described by (5) accurately. Therefore, if the error of the output voltage or current is fed back to affect $u_{\text {modc }}$, the closed-loop control is realized.

Because the voltage and current control strategies are interchangeable, for conciseness, in the following parts of this paper, only the current control strategy is further discussed. To realize the closed-loop control of the current, as mentioned, it is a direct way to assume

$$
u_{\text {modc }}=K e_{\mathrm{i}}=K\left(i_{\mathrm{R}}^{*}-i_{\mathrm{R}}\right)
$$

where $e_{\mathrm{i}}=i_{\mathrm{R}}{ }^{*}-i_{\mathrm{R}}$ is the error of the output current, $i_{\mathrm{R}}{ }^{*}$ is the command current, $K$ is the error amplification and by changing $K$ the degree of the error affecting $u_{\text {modc }}$ is changed. Based on (6) and according to the theory of the similar triangles, the final control quantity of the inverter, i.e., the duty ratio is derived:

$$
D=\frac{t_{\text {on }}}{T_{\mathrm{s}}}=\frac{u_{\mathrm{modc}}+V_{\mathrm{c}}}{2 V_{\mathrm{c}}}=\frac{K e_{\mathrm{i}}}{2 V_{\mathrm{c}}}+\frac{1}{2}
$$

where $V_{\mathrm{c}}$ is the amplitude of the isosceles triangular carrier.

On the basis of all the foregoing discussions, in the last part of this section, the variation of the inductor current over $1 T_{\mathrm{s}}$ (denoted as $\Delta i_{\mathrm{L}}$ ) is analyzed, and some important facts are revealed. According to the theory of calculus, the integration of the current differential $\mathrm{d} i_{\mathrm{L}}$ over $1 T_{\mathrm{S}}$ is approximately equal to the summation of all the small current variations over the same time interval. And the summation of all the small current variations is equal to $\Delta i_{\mathrm{L}}$. Therefore, by integrating the two sides of the Kirchhoff voltage equation in (1) over $1 T_{\mathrm{s}}$, the following expression is obtained:

$$
\begin{gathered}
\Delta i_{\mathrm{L}} \approx \int_{0}^{T_{\mathrm{s}}} \mathrm{d} i_{\mathrm{L}}=\frac{1}{L} \int_{0}^{T_{\mathrm{s}}}\left[(2 p-1) \bar{V}_{\mathrm{dc}}-\left(i_{\mathrm{L}} r+v_{\mathrm{R}}\right)\right] \mathrm{d} t \\
=\frac{1}{L}\left\{\left[-\bar{V}_{\mathrm{dc}}-\left(i_{\mathrm{L}} r+v_{\mathrm{R}}\right)\right] 2 t_{\mathrm{off}}+\left[\bar{V}_{\mathrm{dc}}-\left(i_{\mathrm{L}} r+v_{\mathrm{R}}\right)\right] t_{\mathrm{on}}\right\} \\
=\frac{T_{\mathrm{s}}}{L}\left[(2 D-1) \bar{V}_{\mathrm{dc}}-\left(i_{\mathrm{L}} r+i_{\mathrm{R}} R\right)\right] \\
=\frac{T_{\mathrm{s}}}{L}\left[\frac{K e_{\mathrm{i}} \bar{V}_{\mathrm{dc}}}{V_{\mathrm{c}}}-\left(i_{\mathrm{L}} r+i_{\mathrm{R}} R\right)\right]
\end{gathered}
$$

where it is assumed that the duration of $p=1$ is $t_{\text {on }}$ and that of $p=0$ is $t_{\mathrm{off}}$, and there are 2 durations of $p=0$; here, (7) and the default relation $2 t_{\mathrm{off}}=T_{\mathrm{s}}-t_{\mathrm{on}}$ are also involved in the derivation. Now, as to the last equation of (8), two situations can be discussed. First, let $\Delta i_{\mathrm{L}} \geq 0$ (i.e., $i_{\mathrm{L}}$ increases), and this will give an inequation

$$
e_{\mathrm{i}} \geq \frac{V_{\mathrm{c}}\left(i_{\mathrm{L}} r+i_{\mathrm{R}} R\right)}{K \bar{V}_{\mathrm{dc}}}=e_{\mathrm{i}}^{*} \neq 0
$$

where $e_{\mathrm{i}}^{*}$ is defined to replace the term on the right side of the inequation for conciseness. Second, let $\Delta i_{\mathrm{L}} \leq 0$ (i.e., $i_{\mathrm{L}}$ decreases), and similarly, this will give an inequation as $e_{\mathrm{i}} \leq e_{\mathrm{i}}^{*} \neq 0$. In a word, whether $i_{\mathrm{L}}$ increases or decreases, $e_{\mathrm{i}}$ will always fluctuate around a nonzero value $e_{\mathrm{i}}^{*}$. Therefore, $e_{\mathrm{i}}^{*}$ is named as the inherent tracking error. Due to the existence of $e_{\mathrm{i}}^{*}$, the closedloop control discussed in this section is actually with steadystate errors, and this is the problem that most of the inverters will encounter as well. And the reason why these inverters are not very sensitive to $e_{\mathrm{i}}{ }^{*}$ is that their accuracies have basically met the requirements of their applications, e.g., in different kinds of the flexible AC transmission systems. However, for relay testing, the accuracy is still needed to be promoted, and therefore, $e_{\mathrm{i}}^{*}$ is intolerable.

\section{Proposition of Pseudo-PID Control Method}

In order to counteract $e_{\mathrm{i}}{ }^{*}$, a modified command current, denoted as $\hat{i}_{\mathrm{R}}^{*}$, is defined as 


$$
\hat{i}_{\mathrm{R}}^{*}=i_{\mathrm{R}}^{*}+e_{\mathrm{i}}^{*}=i_{\mathrm{R}}^{*}+\frac{V_{\mathrm{c}}\left(i_{\mathrm{L}} r+i_{\mathrm{R}} R\right)}{K \bar{V}_{\mathrm{dc}}}
$$

Replace the $i_{\mathrm{R}}{ }^{*}$ in $e_{\mathrm{i}}=i_{\mathrm{R}}{ }^{*}-i_{\mathrm{R}}$ with $\hat{i}_{\mathrm{R}}^{*}$ to get the modified error $\hat{e}_{\mathrm{i}}=\hat{i}_{\mathrm{R}}^{*}-i_{\mathrm{R}}$. Then, replace the $e_{\mathrm{i}}$ 's in (7) and the last equation of (8) with $\hat{e}_{\mathrm{i}}$, and reconsider the original relation $e_{\mathrm{i}}=i_{\mathrm{R}}{ }^{*}-i_{\mathrm{R}}$; thus, the modified $D$ and $\Delta i_{\mathrm{L}}$ are obtained:

$$
\begin{gathered}
\hat{D}=\frac{K\left(\hat{i}_{\mathrm{R}}^{*}-i_{\mathrm{R}}\right)}{2 V_{\mathrm{c}}}+\frac{1}{2}=\frac{K e_{\mathrm{i}}}{2 V_{\mathrm{c}}}+\frac{i_{\mathrm{L}} r+i_{\mathrm{R}} R}{2 \bar{V}_{\mathrm{dc}}}+\frac{1}{2} \\
\Delta \hat{i_{\mathrm{L}}} \approx \frac{T_{\mathrm{s}}}{L}\left[\frac{K\left(\hat{i}_{\mathrm{R}}^{*}-i_{\mathrm{R}}\right) \bar{V}_{\mathrm{dc}}}{V_{\mathrm{c}}}-\left(i_{\mathrm{L}} r+i_{\mathrm{R}} R\right)\right]=\frac{T_{\mathrm{s}} K \bar{V}_{\mathrm{dc}} e_{\mathrm{i}}}{L V_{\mathrm{c}}}
\end{gathered}
$$

As to (11), for actual control, it needs to be discretized as

$$
\hat{D}(k)=\frac{K e_{\mathrm{i}}(k)}{2 V_{\mathrm{c}}}+\frac{i_{\mathrm{L}}(k) r+i_{\mathrm{R}}(k) R}{2 \bar{V}_{\mathrm{dc}}}+\frac{1}{2}
$$

where $k=1,2, \cdots$ is the sampling index. And sometimes, the incremental type is more convenient, and it can be derived from $\Delta \hat{D}(k)=\hat{D}(k)-\hat{D}(k-1)$ :

$$
\begin{gathered}
\Delta \hat{D}(k)=\frac{K}{2 V_{\mathrm{c}}}\left[e_{\mathrm{i}}(k)-e_{\mathrm{i}}(k-1)\right]+\frac{r}{2 \bar{V}_{\mathrm{dc}}}\left[i_{\mathrm{L}}(k)-i_{\mathrm{L}}(k-1)\right]+ \\
\frac{R}{2 \bar{V}_{\mathrm{dc}}}\left[i_{\mathrm{R}}(k)-i_{\mathrm{R}}(k-1)\right]
\end{gathered}
$$

As to (12), let $\Delta \hat{i}_{\mathrm{L}} \geq 0$ and $\Delta \hat{\mathrm{i}}_{\mathrm{L}} \leq 0$, and it is seen that $e_{\mathrm{i}}$ fluctuates around 0 now, demonstrating that the inherent tracking error has been totally eliminated. Further, if the coefficient of $e_{\mathrm{i}}$ is assumed to be 1 , a clearer relation is revealed:

$$
\Delta \hat{i_{\mathrm{L}}} \approx e_{\mathrm{i}}
$$

where the constraint condition is

$$
K=\frac{L V_{\mathrm{c}}}{T_{\mathrm{s}} \bar{V}_{\mathrm{dc}}}
$$

Next, some equivalent transformations are carried out on (14) to show its potential details. And to do this, some preparations should be made.

Firstly, discretize (15) under the constraint condition (16):

$$
\Delta \hat{i}_{\mathrm{L}}(k)=i_{\mathrm{L}}(k)-i_{\mathrm{L}}(k-1) \approx e_{\mathrm{i}}(k)
$$

Secondly, discretize the Kirchhoff current equation in (1) with the first-order backward difference:

$$
i_{\mathrm{L}}(k)=\frac{R C}{T_{\mathrm{s}}}\left[i_{\mathrm{R}}(k)-i_{\mathrm{R}}(k-1)\right]+i_{\mathrm{R}}(k)
$$

Thirdly, use (18) to calculate $i_{\mathrm{L}}(k)-i_{\mathrm{L}}(k-1)$, and rearrange it in terms of $i_{\mathrm{R}}(k)-i_{\mathrm{R}}(k-1)$, and then substitute (17) in; thus, the following expression is obtained:

$$
\begin{gathered}
i_{\mathrm{R}}(k)-i_{\mathrm{R}}(k-1) \\
=\left[i_{\mathrm{L}}(k)-i_{\mathrm{L}}(k-1)\right]-\frac{R C}{T_{\mathrm{s}}}\left[i_{\mathrm{R}}(k)-2 i_{\mathrm{R}}(k-1)+i_{\mathrm{R}}(k-2)\right] \\
=e_{\mathrm{i}}(k)-\frac{R C}{T_{\mathrm{s}}}\left[i_{\mathrm{R}}(k)-2 i_{\mathrm{R}}(k-1)+i_{\mathrm{R}}(k-2)\right]
\end{gathered}
$$

Lastly, substitute (17) and (19) into (14), and thus (14) is equivalently transformed to

$$
\begin{aligned}
\Delta \hat{D}(k)= & \frac{K}{2 V_{\mathrm{c}}}\left[e_{\mathrm{i}}(k)-e_{\mathrm{i}}(k-1)\right]+\frac{r+R}{2 \bar{V}_{\mathrm{dc}}} e_{\mathrm{i}}(k)- \\
& \frac{R^{2} C}{2 \bar{V}_{\mathrm{dc}} T_{\mathrm{s}}}\left[i_{\mathrm{R}}(k)-2 i_{\mathrm{R}}(k-1)+i_{\mathrm{R}}(k-2)\right]
\end{aligned}
$$

On the other hand, review the classic formula of PID control method:

$$
u(k)=K_{\mathrm{P}} e(k)+K_{\mathrm{I}} \sum_{j=0}^{k} e(j) T_{\mathrm{s}}+\frac{K_{\mathrm{D}}}{T_{\mathrm{s}}}[e(k)-e(k-1)]
$$

where $u(k)$ is the control quantity, $e(k)$ is the error of the output, $K_{\mathrm{P}}, K_{\mathrm{I}}$ and $K_{\mathrm{D}}$ are the proportional, integral and derivative parameters respectively, and the definitions of $k$ and $T_{\mathrm{s}}$ are the same as the above. Similarly, the incremental type of (21) can be derived from $\Delta u(k)=u(k)-u(k-1)$ :

$$
\Delta u(k)=K_{\mathrm{P}}[e(k)-e(k-1)]+K_{\mathrm{I}} T_{\mathrm{s}} e(k)+
$$

$$
\frac{K_{\mathrm{D}}}{T_{\mathrm{s}}}[e(k)-2 e(k-1)+e(k-2)]
$$


Now, compare (20) with (22), and it is seen that the previous two terms of the two formulae are corresponding to each other one to one, however, the third term of (20) is composed of load currents and that of (22) is composed of errors. In a word, (20) is similar but not identical to (22). Therefore, the control method represented by (20) is not real PID control method. And due to this, (20) is named as pseudo-PID control method. Also, from comparison, three formulae can be obtained for calculating the pseudo-PID parameters:

$$
\left\{\begin{array}{l}
K_{\mathrm{P}}=\frac{K}{2 V_{\mathrm{c}}}=\frac{L}{2 T_{\mathrm{s}} \bar{V}_{\mathrm{dc}}}, \\
K_{\mathrm{I}}=\frac{r+R}{2 T_{\mathrm{s}} \bar{V}_{\mathrm{dc}}}, \\
\hat{K}_{\mathrm{D}}=-\frac{R^{2} C}{2 \bar{V}_{\mathrm{dc}}},
\end{array}\right.
$$

where the pseudo-D parameter is denoted as $\hat{K}_{\mathrm{D}}$ to be distinguished from $K_{\mathrm{D}}$. Based on (23), (20) can be simplified as

$$
\begin{gathered}
\Delta \hat{D}(k)=K_{\mathrm{P}}\left[e_{\mathrm{i}}(k)-e_{\mathrm{i}}(k-1)\right]+K_{\mathrm{I}} T_{\mathrm{s}} e_{\mathrm{i}}(k)+ \\
\frac{\hat{K}_{\mathrm{D}}}{T_{\mathrm{s}}}\left[i_{\mathrm{R}}(k)-2 i_{\mathrm{R}}(k-1)+i_{\mathrm{R}}(k-2)\right]
\end{gathered}
$$

\section{DISCUSSIONS ON PSEUDO-PID PARAMETERS}

In this section, the pseudo-PID parameters are further discussed by two groups of comparisons, and the features and advancement of the pseudo-PID control method are revealed.

Firstly, the formulae of the pseudo-PID parameters, i.e. (23), are compared with the empirical formulae of the PI parameters which are widely used in the closed-loop control of inverters and were originally proposed in [17].

For clarity, the symbols of the empirical formulae of [17] are equivalently replaced with the ones used in this paper, and the empirical formulae are rewritten as follows:

$$
\left\{\begin{array}{l}
K_{\mathrm{P}}^{\prime}=\frac{L}{2 T_{\mathrm{s}} \bar{V}_{\mathrm{dc}}}, \\
K_{\mathrm{I}}^{\prime}=\frac{L}{2 T_{\mathrm{s}}^{2} \bar{V}_{\mathrm{dc}}},
\end{array}\right.
$$

where an apostrophe is added on $K_{\mathrm{P}}$ and $K_{\mathrm{I}}$ for distinguishing. Based on plenty of simulations, a conclusion was drawn in [17] that with the PI parameters in (25) the inverter presented good dynamic performance, i.e., quick current response and relatively high accuracy of the output current.
From the comparison of (23) and (25), it is seen that $K_{\mathrm{P}}^{\prime}=$ $K_{\mathrm{P}}$, however, if $K_{\mathrm{I}}^{\prime}=K_{\mathrm{I}}$ is required, the relation $L / T_{\mathrm{s}}=r+R$ should be fulfilled. And to confirm this, the units and values of both sides should be compared. First, compare the units. When the angular frequency, i.e., $\omega_{\mathrm{s}}=2 \pi / T_{\mathrm{s}}$, is introduced, $L / T_{\mathrm{s}}$ can be transformed to $\omega_{\mathrm{s}} L /(2 \pi)$, showing that the unit of $L / T_{\mathrm{s}}$ is actually $\Omega$, which is the same as $r+R$. So the key point is to compare the values. From the analysis of the actual application situations, it is found that the values of $L / T_{\mathrm{s}}$ and $r+R$ are actually close to each other. Here, the inverter that is designed for relay testing in this paper and the inverter presented in [17] are taken as examples to validate this argument. As to the former, the hardware parameters are listed in Appendix, and calculation gives that $L / T_{\mathrm{s}}=18 \Omega$ and $r+R=19.4 \Omega$; as to the latter, the hardware parameters are $L=13 \mathrm{mH}, T_{\mathrm{s}}=6.67 \times 10^{-4} \mathrm{~s}, r=20 \Omega$, $R=2 \Omega$, and calculation gives that $L / T_{\mathrm{s}}=19.5 \Omega$ and $r+$ $R=22 \Omega$. Therefore, the reason why [17] found $K_{\mathrm{P}}^{\prime}$ and $K_{\mathrm{I}}^{\prime}$ appropriate and effective is that they had matched $K_{\mathrm{P}}$ and $K_{\mathrm{I}}$ to a great degree, i.e., $K_{\mathrm{P}}^{\prime}=K_{\mathrm{P}}$ and $K_{\mathrm{I}}^{\prime} \approx K_{\mathrm{I}}$. However, the accurate value of $K_{\mathrm{I}}$ should be given by (23).

Secondly, the effects of the three pseudo-PID parameters on the control quantity are compared with each other.

Although the values of the pseudo-PID parameters are given by (23), their final effects on the control quantity are reflected in (24), where $K_{\mathrm{I}}$ and $\hat{K}_{\mathrm{D}}$ should be considered as $K_{\mathrm{I}} T_{\mathrm{s}}$ and $\hat{K}_{\mathrm{D}} / T_{\mathrm{S}}$ except $K_{\mathrm{P}}$. And with the hardware parameters listed in Appendix, the corresponding numerical values can be calculated, and the results are: $K_{\mathrm{P}}=0.1343, K_{\mathrm{I}} T_{\mathrm{s}}=0.1448, \hat{K}_{\mathrm{D}} / T_{\mathrm{S}}=-$ 0.0253 . It is seen that $\hat{K}_{\mathrm{D}} / T_{\mathrm{s}}$ is about 5 times smaller than $K_{\mathrm{P}}$ and $K_{\mathrm{I}} T_{\mathrm{s}}$. Therefore, abandoning the third term of (24), which reduces the pseudo-PID control method to PI control method, has no great effect on the whole control but certain effect on the accuracy of the output current. On the other hand, if the third term of (22) is used instead of the third term of (24), i.e., the pseudo-PID control method is turned into PID control method, this is equivalent to introducing a disturbance term to the control quantity due to the lack of theoretical basis, and its effect on the control quantity is relevant to $K_{\mathrm{D}} / T_{\mathrm{s}}$. When $K_{\mathrm{D}} / T_{\mathrm{s}}$ is relatively large, the effect of the third term will be notable, and thus the system is likely to incur oscillations. However, when $K_{\mathrm{D}} / T_{\mathrm{s}}$ is relatively small, the effect of the third term is limited, and thus the system is unlikely to be affected. This explains why the introduction of D parameter will sometimes greatly affect the stability of the system, but sometimes seem to have little effect on the system. And, this also demonstrates that PID control method is unsuitable for the closed-loop control of the PWM inverters, and explains why it is barely adopted.

In short, the third term of (24) serves as an extra dynamic compensator, if correctly used, i.e., $\hat{K}_{\mathrm{D}}$ is adjusted according to (23), it can compensate the inherent tracking error and thus promote the accuracy of the output current, otherwise it is likely to harm the stability of the system. 


\section{SiMULATION AND EXPERIMENTAL VERIFICATIONS}

In order to verify the performance of the pseudo-PID control method, in this section, it is applied to a single-phase PWM inverter for relay testing, the hardware parameters of which are listed in Appendix A, and the results are presented by two groups of comparative examples.

Firstly, through simulation and experiment, the performance of the pseudo-PID control method is compared with that of PI control method (because PID control method is not applicable as verified) to show the promotion of the accuracy of the output current. Here, the software simulation is carried out by MATLAB programs directly on the basis of the discrete type of (1) together with (24), as is simpler than by MATLAB SIMULINK. And the experiment is carried out on the singlephase PWM inverter shown in Figure 1. Then, the waveforms of the output current produced by simulation and experiment are both presented in Figure 3 for comparison, and obviously, they are in good agreement.

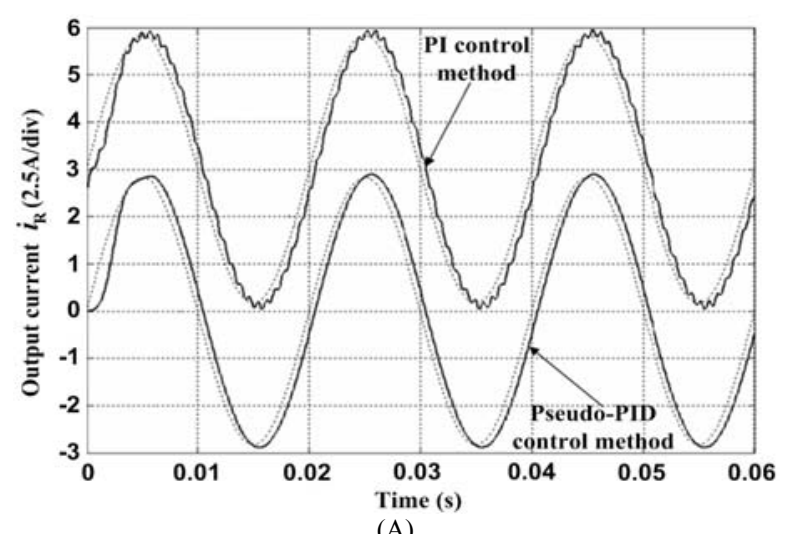

(A)

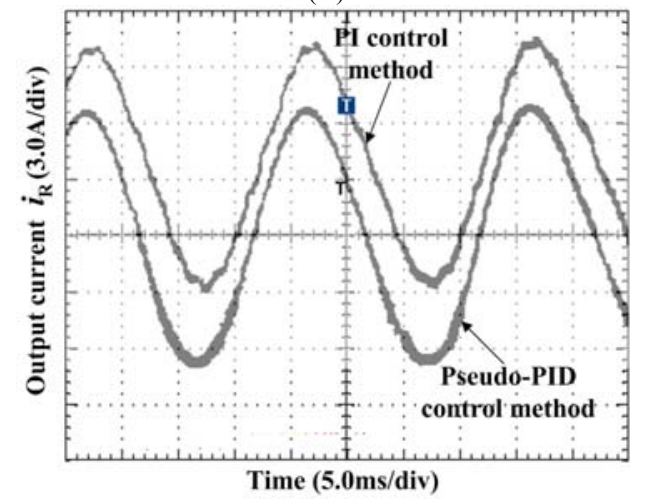

(B)

FIGURE III. COMPARISON OF PSEUDO-PID CONTROL METHOD AND PI CONTROL METHOD: (A) SIMULATION WAVEFORMS, (B) EXPERIMENTAL WAVEFORMS

From Figure 3, it is seen that the simulation and experimental waveforms produced by PI control method fluctuate around the command current continuously, illustrating the existence of the inherent tracking error; however, those produced by the pseudo-PID control method are very stable and smooth, illustrating that the small fluctuations and distortions of the waveforms caused by the inherent tracking error are compensated. Therefore, the comparison demonstrates qualitatively that the pseudo-PID control method has inhibited the inherent tracking error effectively, and thus promotes the accuracy of the output current.

Secondly, through experiment, the single-phase PWM inverter (based on the pseudo-PID control method) is com-pared with the LPA to assess its accuracy level. The experiment is respectively carried out on the single-phase PWM inverter and the current-type LPA of a general-purpose relay testing device, and for comparison they are both used to re-produce the same fault current that is recorded by the digital fault recorder. It is necessary to point out here that reproducing the fault current/voltage is a novel and important function of the modern relay testing device, which can provide the relay or automation equipment under test with "real" fault signals [18-20]. And, the fault current shown in Figure 4(a) is taken as an example for reproducing. Because the waveforms of the output current reproduced by the single-phase PWM inverter and the LPA are both very like the one in Figure 4(a) and it is already difficult to distinguish the differences from appearance, for conciseness, only the wave-form of the output current reproduced by the single-phase PWM inverter is presented (Figure 4(b)).
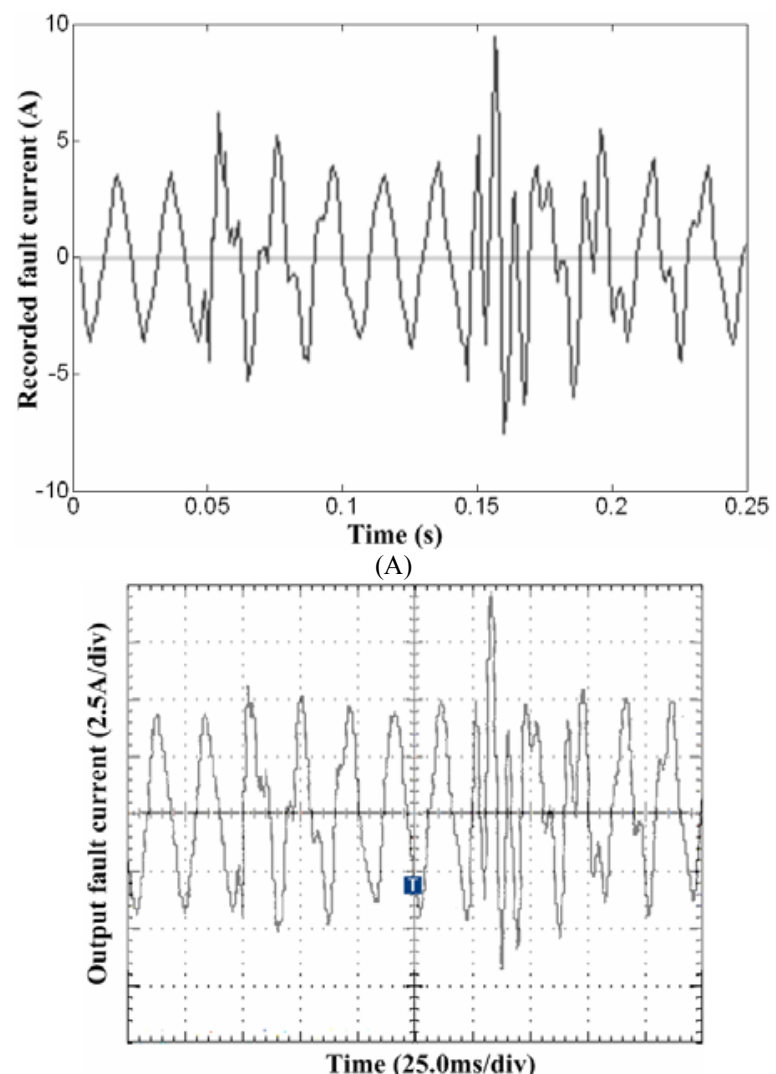

(B)

FIGURE IV. FAULT CURRENT REPRODUCING EXPERIMENT: (A) FAULT CURRENT RECORDED BY DIGITAL FAULT RECORDER, (B) FAULT CURRENT REPRODUCED BY SINGLE-PHASE PWM INVERTER WITH PSEUDO-PID CONTROL

Also because of the great likeness between the original fault current and the reproduced ones, the accuracy of the output current can no longer be assessed qualitatively, but has to be assessed quantitatively, where the root mean square error (RMSE) is chosen as the assessment criterion. The reason to 
choose the RMSE is that it can go deep into the waveform to assess the point-to-point errors, and this feature is shown by formula

$$
\varepsilon_{\mathrm{RMSE}}=\sqrt{\frac{1}{\mathrm{~N}} \sum_{k=1}^{\mathrm{N}}\left[i_{\mathrm{R}}^{*}(k)-i_{\mathrm{R}}(k)\right]^{2}}=\frac{\left\|\boldsymbol{i}_{\mathrm{R}}^{*}-\boldsymbol{i}_{\mathrm{R}}\right\|_{2}}{\sqrt{\mathrm{N}}}
$$

where $\mathrm{N}$ is the number of the sampled values, $\boldsymbol{i}_{\mathrm{R}}^{*}$ is the command current vector, $\boldsymbol{i}_{\mathrm{R}}$ is the load current vector, and $\|\cdot\|_{2}$ denotes the 2-norm calculation. According to (26), the experimental results are assessed quantitatively as follows: as to the LPA, $\varepsilon_{\text {RMSE }}=0.0372$; as to the single-phase PWM inverter, $\varepsilon_{\mathrm{RMSE}}=0.0415$; the deviation of the two is 0.0043 . It is apparent that with the aid of the pseudo-PID control method the accuracy of the output current of the single-phase PWM inverter is already comparable to that of the LPA. Therefore, based on the pseudo-PID control method, the single-phase PWM inverter can now replace the LPA for relay testing, and due to its higher efficiency and simpler structure it can make the relay testing device more cost effective.

\section{CONCLUSION}

Through an in-depth analysis of the operation and control processes of the single-phase PWM inverter, it is verified that PID control method is unsuitable for the closed-loop control of the PWM inverters, and therefore it is barely adopted. And the analytical process has revealed the existence of the inherent tracking error. To counteract the inherent tracking error, a modified command current is introduced, and this gives birth to the pseudo-PID control method that is similar but not identical to PID control method. Together, three useful formulae are produced for calculating the pseudo-PID parameters. Because of the solid theoretical basis, the first two formulae are more accurate than the widely used empirical formulae of PI parameters. And the third formula provides a term that serves as an extra dynamic compensator of the control quantity, which can compensate the inherent tracking error and thus promotes the accuracy of the output current. Finally, the pseudo-PID control method is applied to a single-phase PWM inverter for relay testing, and the simulation and experimental results demonstrate that with the aid of the pseudo-PID control method the accuracy of the output current of the inverter is already comparable to that of the LPA, and therefore, the single-phase PWM inverter can now replace the LPA for relay testing purpose and thus makes the relay testing device more cost effective. Due to the fundamentality and generality of the pseudo-PID control method, it can be conveniently introduced to complex inverters for performance promotion.

\section{APPENDIXA}

AC power source: $220 \mathrm{~V}(\mathrm{RMS})$; rectifier/isolation transformer: 220/100 V(RMS); rectifier bridge: KBPC5002; IGBT module/intelligent power module: PM30CSJ060; fast-recovery free-wheeling diode: HFA04TB60; $C_{\mathrm{d}}: 4700 \mu \mathrm{F} ; \bar{V}_{\mathrm{dc}}: 67 \mathrm{~V} ; L$ : $1.8 \mathrm{mH} ; C: 37.6 \mu \mathrm{F} ; r: 16.4 \Omega ; R: 3 \Omega ; T_{\mathrm{s}}: 1 \times 10^{-4} \mathrm{~s}$; digital signal processor (DSP): TMS320LF2407A.

\section{ACKNOWLEDGMENT}

This work is supported by the Science and Technology Project of Chongqing Education Committee (KJ1603 605).

\section{REFERENCES}

[1] Y. J. He, J. J. Liu, Z. A. Wang, Y. P. Zou, “A PI control algorithm with zero static misadjustment for tracking the harmonic current of threelevel APFs," Journal of Power Electronics, vol. 14, no. 1, pp. 175-182, 2014.

[2] T. L. Tiang, D. Ishak, "Modeling and simulation of deadbeat-based PI controller in a single-phase H-bridge inverter for stand-alone applications," Turkish Journal of Electrical Engineering and Computer Sciences, vol. 22, no. 1, pp. 43-56, 2014.

[3] J. K. Li, J. Q. Wang, Y. Xu, J. T. Li, J. J. Chen, P. F. Hou, et al, "Research on discretization PI control technology of single-phase gridconnected inverter with LCL filter," Mathematical Problems in Engineering, vol. 2014, no. 1, pp. 70-76, 2014.

[4] P. Wisutmetheekorn, V. Chunkag, "DSP based control implementation of an AC/DC converter with improved input current distortion," Advances in Electrical and Computer Engineering, vol. 11, no. 2, pp. 8794, 2011.

[5] H. Takaaki, Y. Kou, H. Huo, "The parameterization of all plants stabilized by a PID controller for minimum phase system," ICIC Express Letters, vol. 9, no. 5, pp. 1525-1531, 2015.

[6] Y. Bo, D. W. Sun, "Fuzzy PID control technology for synchronous generator excitation," International Journal of Control and Automation, vol. 8, no. 10, pp. 91-98, 2015.

[7] T. Geng, Y. F. Lv, Y. Liu, "Expert self-tuning using fuzzy reasoning for PID controller," Communications in Computer and Information Science, vol. 472, pp. 138-141, 2014.

[8] K. Laroussi, M. Zelmat, M. Rouff, "Implementation of a fuzzy logic system to tune a PI controller applied to an induction motor," Advances in Electrical and Computer Engineering, vol. 9, no. 3, pp. 107-113, 2009.

[9] M. J. Mahmoodabadi, M. Taherkhorsandi, M. Talebipour, K. K. Castillo-Villar, "Adaptive robust PID control subject to supervisory decoupled sliding mode control based upon genetic algorithm optimization," Transactions of the Institute of Measurement and Control, vol.37, no. 4, pp. 505-514, 2015.

[10] M. B. Milovanović, D. S. Antić, M. T. Milojković, S. S. Nikolić, S. L. Perić, M. D. Spasić, "Adaptive PID control based on orthogonal endocrine neural networks," Neural Networks, vol. 84, pp. 80-90, 2016.

[11] O. T. Altinoz, H. Erdem, "Particle swarm optimisation-based PID controller tuning for static power converters," International Journal of Power Electronics, vol. 7, no. 1-2, pp. 16-35, 2015.

[12] M. Dangor, O. A. Dahunsi, J. O. Pedro, M. M. Ali, "Evolutionary algorithm-based PID controller tuning for nonlinear quarter-car electrohydraulic vehicle suspensions," Nonlinear Dynamics, vol. 78, no. 4, pp. 2795-2810, 2014.

[13] N. Katal, S. K. Singh, "Multi-objective optimization of PID controller for DC servo motor using genetic algorithm," Engineering Intelligent Systems, vol. 23, no. 1, pp. 7-16, 2015.

[14] X. M. Sun, H. L. Long, D. C. Liu, "Multi-objective optimization design for snubber in switching power amplifier," in Proceedings of the 12th International Conference on Electrical Machines and Systems, Tokyo, 2009, pp. 1-6.

[15] B. Hoff, W. Sulkowski, "Grid-connected VSI with LCL filter - models and comparison," IEEE Transactions on Industry Applications, vol. 50, no. 3, pp. 1974-1981, 2014.

[16] D. Ankan, B. Subhashish, "Control of dynamic VAR compensator based on current source converter," in Proceedings of the 7th Annual IEEE Energy Conversion Congress and Exposition, 2015, pp. 3442-3448.

[17] J. Dixon, S. Tepper, L. Morán, "Practical evaluation of different modulation techniques for current-controlled voltage source inverters," IEE Proceedings: Electric Power Applications, vol. 143, no. 4, pp. 301$306,1996$. 
[18] X. M. Sun, D. C. Liu, H. L. Long, L. Yun, "The design of an electrical fault-waveform regenerator," in Proceedings of the 11th International Conference on Electrical Machines and Systems, Wuhan, 2008, pp. 136141.

[19] M. O. Faruque, T. Strasser, G. Lauss, V. Jalili-Marandi, C. Dufour, P. Forsyth, et al, "Real-time simulation technologies for power systems design, testing and analysis," IEEE Power and Energy Technology Systems Journal, vol. 2, no. 2, pp. 63-73, 2015.

[20] A. Botezan, R. Tirnovan, R. Munteanu, A. Graur, D. Rafiroiu, "Hysteresis current control of the single-phase voltage source inverter using eMEGAsim real-time simulator," Advances in Electrical and Computer Engineering, vol. 15, no. 3, pp. 99-104, 2015. 